common imatinib-target mutation was in KIT exon 11, followed by KIT exon 9 and KIT exon 17. The results showed that the KIT exon 11 mutation had a favorable effect on objective response, time to tumor progression and overall survival compared with the exon $9 \mathrm{KIT}$ mutation and wild-type genotypes. The dose of imatinib did not affect survival outcomes for patients with exon-9, exon-11 or wild-type mutations. A significantly higher response rate was observed in patients treated with the higher imatinib dose compared with the lower dose in those with exon 9 KIT mutations.

The authors conclude that these results confirm the positive effect of exon 11 KIT mutations on patients treated with imatinib for advanced GIST.

Original article Heinrich MC et al. (2008) Correlation of kinase genotype and clinical outcome in the North American Intergroup phase III trial of imatinib mesylate for treatment of advanced gastrointestinal stromal tumor: CALGB 150105 study by Cancer and Leukemia Group B and Southwest Oncology Group. J Clin Oncol 26: 5360-5367

\section{HER2 and EGFR status and correlation with response to lapatinib and chemotherapy}

Lapatinib inhibits HER2 and EGFR, but which patients with breast cancer are most likely to benefit from this treatment is unclear. Press et al. assessed the DNA, mRNA and protein level status of HER2 and EGFR in women with metastatic breast cancer who were treated with lapatinib and chemotherapy in two randomized, phase III trials, and correlated the data with clinical outcome to determine associations between clinical response and HER2 and EGFR status.

Fluorescent in situ hybridization, reverse transcription polymerase chain reaction and immunohistochemistry were used to assess HER2 and EGFR gene, mRNA and protein levels, respectively. HER2 amplification was detected in 344 of 733 women (47\%), and strong HER2 protein staining was seen in 279 of 798 samples assessed (35\%). A significant correlation between HER2 amplification and HER2 protein expression existed. EGFR expression correlated with mRNA levels, but no correlation between HER2 amplification or gene expression and EGFR status was observed. A clinical improvement was noted in HER2-positive patients who received lapatinib and chemotherapy compared with those treated with chemotherapy alone. No correlation between EGFR status and responsiveness to lapatinib was noted, regardless of HER2 status. Although women with HER2-negative tumors who received combination therapy had a prolonged progression-free survival, these results were not confirmed by an independent research laboratory.

The authors conclude that women with HER2-positive metastatic breast cancer benefit from lapatinib, but women with HER2-negative tumors experience no benefit.

Original article Press MF et al. (2008) HER-2 gene amplification, HER-2 and epidermal growth factor receptor mRNA and protein expression, and lapatinib efficacy in women with metastatic breast cancer. Clin Cancer Res 14: 7861-7870

\section{COX2 and EGFR variants that predict survival in colorectal cancer}

A study has identified independent genetic markers of survival in patients with metastatic colorectal cancer treated with the anti-EGFR antibody cetuximab. Overexpression of EGFR, and mutations in the EGFR-activating KRAS oncogene, are associated with poor prognosis in patients with colorectal cancer. Lurje et al. have found that polymorphisms in the cyclooxygenase 2 (COX2) and EGFR genes predict progression-free survival (PFS) independently of KRAS status in patients with metastatic colorectal cancer.

The investigators analyzed tissue samples from 130 participants in the IMC-0144 trial of cetuximab. Eleven polymorphisms in eight genes associated with the EGFR signaling pathway were assessed for their influence on clinical outcome. Four polymorphisms - two in COX2, one in EGF and one in EGFR-significantly affected PFS, and two of these (COX2$765 \mathrm{G}>\mathrm{C}$ and $E G F R+497 \mathrm{G}>\mathrm{A}$ ) predicted PFS independently of KRAS status, skin reaction to cetuximab, and Eastern Cooperative Oncology Group performance status. COX2-765 C/C homozygotes had median PFS and overall survival of 5.8 months and 10.5 months, respectively, compared with 1.3 months and 5.5 months or less for those carrying the $G$ allele. EGFR $+497 \mathrm{G} / \mathrm{A}$ heterozygotes had 